\title{
Review of the Book Merging the Instructional Design Process With Learner-Centered Theory-The Holistic 4D Model, by Charles M. Reigeluth and Yunjo An
}

Reviewed by Sunnie Lee Watson, $\mathrm{PhD}$

Purdue University, West Lafayette, Indiana, United States

iD https://orcid.org/oooo-0003-2998-9900

Contact: watson82@purdue.edu

Keywords: instructional design, Holistic $4 D$ model, book review

Date Submitted: April 9, 2021 | Date Published: September 16, 2021

\section{Recommended Citation}

Watson, S. L. (2021). Review of the book Merging the Instructional Design Process With Learner-Centered Theory-The Holistic 4D Model, by Charles M. Reigeluth and Yunjo An. Journal of Educational Research \& Practice 11 (1), 217-219. https://doi.org/10.5590/JERAP.2021.11.1.15

\section{Review}

Reigeluth, C. M., \& An, Y. (2020). Merging the instructional design process with learner-centered theory: The Holistic $4 \mathrm{D}$ model. Routledge.

As an instructional designer and faculty member who researches and teaches about the instructional design (ID) process, I know first-hand that learning design and instructional systems design are often taught to and understood by novices as rather fragmented approaches. Merging the Instructional Design Process with Learner-Centered Theory-The Holistic 4D Model explores how to bring together the innovations of these processes, merging instructional design and learning theory into a modern ID process that is better suited to the complex demands of modern instructional contexts.

Written by Dr. Charles Reigeluth, professor emeritus of Indiana University and a towering figure in the field for his work on envisioning and realizing learner-centered paradigms of instruction, and his former student, Dr. Yunjo An, an associate professor who conducts notable research on integrating learner-centered technologies, including educational games, this textbook demands a place in the classrooms of ID programs around the world and a space on the shelf of practitioners. It offers the next generation of instructional designers a new lens that looks beyond the basics of learning design and development, helping them to successfully respond to the shifts of today's learning landscape. Specifically, this book will be helpful to both novice and experienced instructional designers, as it will help guide their design efforts, as well as to teachers, instructors, and trainers interested in incorporating more learner-centered instructional experiences for their students.

The book starts with an introduction and then moves on to illustrate the four major processes of the Holistic 4D model; Define, Design, Develop, and Deploy. Chapter 1 provides an introductory chapter that addresses what instructional design is, what kinds of skills are needed for doing good instructional design, and an 
overview of the most well-known ID models, as well as the Holistic 4D Model itself. The Holistic 4D Model focuses on design for learner-centered instruction; targets both training and educational contexts; allows for more creative and flexible design processes, thereby increasing both efficiency and effectiveness; incorporates instructional theory to guide the selection of methods; and incorporates frequent, formative evaluations throughout, among other advantages. The rest of the text goes into detail about each of the four processes, which are implemented through three stages: top-level, mid-level, and lower-level, with each containing its own cycles of analysis, design, and evaluation.

Chapter 2 details the "Define" process of the model. It describes activities that are important before beginning the ID process, such as performance analysis, needs assessment, project planning, and instructional system planning.

Then the book proceeds to the "Design" section, which is the longest section of the book, spanning Chapter 3 to Chapter 8. In Chapter 3, the authors explore the Design phase of the ID process, introducing the readers to categories of learning. The chapter explains the importance of determining which methods are most effective for a given instructional situation. A description of behavioral, cognitive, and constructivist learning theories and seminal instructional theories are also provided in this chapter.

Chapter 4 discusses nine major considerations for an IDer to bear in mind during the instructional design process, such as the difference between task and topic expertise, the various kinds of analyses that are needed, the importance of just-in-time analysis, the role of subject-matter experts, rapid prototyping, the use of design documents, learner-centered and teacher-centered approaches in instruction, strategies for increasing motivation, and the selection of appropriate educational media.

Chapters 5 and 6 offer detailed guidance for three major, top- and mid-level ADE (analysis, design, and evaluation) events within the Holistic 4D Model. Chapter 5 focuses on the top level where an IDer creates a "fuzzy vision" of the instruction, which includes a high-level outlook and plan for the content, sequence, and instructional methods, formative evaluation, and revisions. Chapter 6 details the eight major mid-level ADE events. This is where the IDer creates further clarity about the vision, content, sequence, objectives, assessments, instructional methods, and media.

Chapter 7 further details guidance for lower-level ADE events of the Holistic 4D model. This is where the IDer is providing final clarity of the vision, including content, sequence, and methods on task and topic expertise. The final chapter of the "Design" phase, Chapter 8, provides advice for designing just-in-time learning. It also provides recommendations on formative evaluation and revision strategies in the process. Finally, it provides suggestions for developing implementation plans and revising plans for project management.

The third section of the Holistic 4D Model, "Develop," details developing the instruction and conducting formative evaluation. Chapter 9 delivers major considerations and detailed guidance for producing instructional media, scriptwriting and storyboarding, graphic design, developing technology-enhanced instruction, and developing a learner guide and an instructor guide. This chapter emphasizes the importance of frequent evaluation and revisions within the development phase. Chapter 10 specifies considerations and recommendations for conducting continuing formative evaluation of the design as well as the ID process. The connections of these elements throughout the entire book demonstrate the iterative and recursive characteristics of the Holistic 4D model.

The final "Deploy" section of the Holistic 4D Model is explored in Chapter 11, where the authors provide recommendations and guidelines for ensuring that the delivery, management, and support functions of an instructional system are operating in a satisfactory manner. Chapter 12 concludes the book by providing detailed advice and guidance in managing a summative evaluation of the instructional system through the Holistic 4D Model lens. 
The text offers a number of strengths that improve its utility, as well as a few areas that could be improved. It is clear that the book was written with a strong focus on being useful and useable. This is reflected in several ways. First, the book is well referenced with some chapters including dozens of references to clarify and support its ideas. Additionally, to support its use as a textbook, the chapters provide suggested exercises to help students better break down and analyze the ideas. Finally, the appendix consolidates every form and template provided within the book so that they may be photocopied to be used in conjunction with the offered exercises or application to individual courses or professional projects. A link to electronic versions is also provided for convenience.

The inclusion of these components not only offers significant benefit to professors and their students using the text, but also to practitioners seeking to apply the Holistic 4d Model to their own work. These strengths could have been enhanced with a few additional elements, such as a glossary. A glossary could be helpful to novices in the field familiarizing themselves with certain concepts and terms for the first time, as well as to veterans who would benefit from the clarity regarding terms whose meaning and use in the field shift over time. Additionally, a complete list of references at the end of the book could provide professors and researchers an easier way to refer back to a reference of interest without having to locate the chapter that included it. Finally, the inclusion of a sample instructional design case to demonstrate the application of the concepts and strategies presented in each chapter could help improve understanding; although, individual, unconnected examples are provided for clarity throughout the chapters. Ultimately, despite the few aspects that could perhaps be improved, overall, the book shows that the authors applied considerable care, resulting in effective and efficient presentation of important ideas and considerable support for readers regardless of the ID context and goals they bring to the text.

As an instructor of practicing and future instructional designers as well as a former corporate instructional designer, I highly recommend this textbook as a foundational text for bridging the misconceptions and fractured approaches that both novice and experienced instructional designers often bring to their work. While our more traditional ID models seek to clearly present themselves as modernized, iterative, and reflexive processes that are suitable for the complexities of current instructional and training contexts, too often it is the fractured, sequential, waterfall ADDIE (Analysis, Design, Development, Implementation, and Evaluation) view that remains at the core of what novice and experienced designers alike bring to their practice.

This text visualizes a holistic approach in a concise and easy-to-understand format that will serve to conceptualize processes that are truly agile, responsive, realistic, and centered on concepts of learnercenteredness, effectiveness, and efficiency and meet the needs of those in all instructional and training contexts. It both presents a look at the history of the field and situates itself within the current and future contexts and needs of our increasingly complex practice. Student and professional alike will find much within its covers to inform and guide their practice.

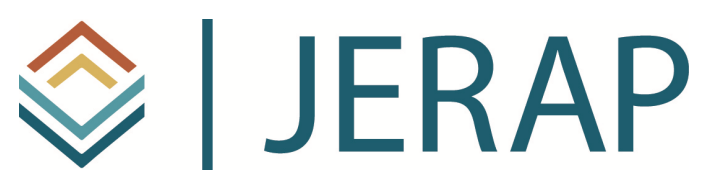

The Journal of Educational Research and Practice is a peerreviewed journal that provides a forum for studies and dialogue about developments and change in the field of education and learning. The journal includes research and related content that examine current relevant educational issues and processes. The aim is to provide readers with knowledge and with strategies to use that knowledge in educational or learning environments. JERAP focuses on education at all levels and in any setting, and includes peer-reviewed research reports, commentaries, book reviews, interviews of prominent individuals, and reports about educational practice. The journal is sponsored by The Richard W. Riley College of Education and Leadership at Walden University, and publication in JERAP is always free to authors and readers. 